\title{
Interferon- $\gamma$ and interleukin-4 detected in serum and saliva from patients with oral lichen planus
}

\author{
Wen-Zhao Liu ${ }^{1,2,3}$, Ming-Jing $\mathrm{He}^{3}$, Long Long ${ }^{3}$, Dong-Liang $\mathrm{Mu}^{3}$, Ming-Shu Xu ${ }^{3}, \mathrm{Xue} \mathrm{Xing}^{3}, \mathrm{Xin} \mathrm{Zeng}^{3}, \mathrm{Ga} \mathrm{Liao}^{3}$, \\ Hong-Xia $\operatorname{Dan}^{3}$ and Qian-Ming Chen ${ }^{3}$
}

\begin{abstract}
Our previous salivary study had demonstrated an apparent T helper 2 (Th2)-predominance in saliva of oral lichen planus (OLP) patients and suggested a potential of salivary interleukin-4 (IL-4) as a biomarker for monitoring disease severity. To further determine the consistency of Th1/Th2 bias of OLP, this study investigated the expression profile of interferon- $\gamma$ (IFN- $\gamma$ ) and IL-4 in serum and the relationship of the serum levels of these cytokines with their saliva partners. Sixty ethnic Chinese patients with OLP (40 of the erythematous/ulcerative form and 20 of the reticular form) were recruited for this study, with 40 age-sex-matched healthy volunteers as control group. IFN- $\gamma$ and IL-4 levels in serum and paired saliva samples were screened by enzyme-linked immunosorbent assay. OLP patient showed a low-level IFN- $\gamma$ but high-level IL-4 expression profile in both serum and saliva, with a lower IFN- $\gamma /$ IL- 4 ratio. Serum IL-4 level in the erythematous/ulcerative group was significantly higher than that in the reticular group. Serum levels of IFN- $\gamma$ and IL-4 were significantly and positively correlated with their saliva partners. These results provided more evidence for Th2 cytokinepredominant immune imbalance in OLP, as well as the potential of IL-4 as the biomarker for monitoring severity of OLP.
\end{abstract} International Journal of Oral Science (2013) 6, 22-26; doi:10.1038/ijos.2013.74; published 25 October 2013

Keywords: oral lichen planus; saliva; serum; T helper cytokines

\section{INTRODUCTION}

The signature cytokines of distinct T-cell subsets and the transcriptional regulation of T-cell differentiation are indicated to be of fundamental importance in mucosal immunity. ${ }^{1}$ A key component of mucosal immune response is mediated by $\mathrm{CD} 4^{+} \mathrm{T}$ lymphocytes that can differentiate into functionally distinct subsets. Whereas $\mathrm{T}$ helper 1 (Th1) cells secrete the cytokines interferon- $\gamma($ IFN- $\gamma$ ) and tumor-necrosis factor- $\alpha$, Th2 cells secrete interleukin- 4 (IL-4), IL-5, IL-9 and IL13. The mutually antagonistic effects of IFN- $\gamma$ and IL-4, the principal cytokines of Th1 and Th2 cells respectively, regulate Th1/Th2 balance and subsequent polarization. ${ }^{2}$ A close relationship between Th1/Th2 imbalance and pathogenesis of mucosal inflammatory diseases has been well demonstrated, such as Th2-predominence in allergic asthma $^{3}$ and Th1-predominence in Crohn's disease. ${ }^{4}$ Skewing of Th1/Th2 balance is critical for the clinical outcome and may become a new therapeutic strategy for immunologic disorders. ${ }^{4-6}$

Oral lichen planus (OLP) is a chronic inflammatory oral mucosal disease characterized by a $\mathrm{T}$ lymphocyte-mediated immune response, affecting about $0.5 \%-2 \%$ of the world population and persisting up to more than 20 years without spontaneous remission. ${ }^{7-8}$ Th1 and Th2 type cytokines in OLP, especially IFN- $\gamma$ and IL-4, have been studied extensively with several research media, ${ }^{9-14}$ but the conclusions of these studies were quite controversial, probably due to the small sample size. Our previous study with relatively large sample size demonstrated a Th2-predominant expression profile in saliva of OLP patients. ${ }^{15}$ To further determine the systemic Th1/Th2 expression pattern and the consistency of Th1/Th2 bias between saliva and serum in OLP, we performed the current study, investigating the expression profile of IFN- $\gamma$ and IL-4 in sera and saliva simultaneously collected from the OLP patients and healthy controls.

\section{MATERIALS AND METHODS}

Subjects

A sum of 60 ethnic Chinese patients (female/male: 35/25; mean age: $45 \pm 16$ years old) clinically and histopathologically diagnosed as OLP, were recruited in this study, according to clinical classification ${ }^{7}$ and the definition of OLP by the World Health Organization. Forty of the OLP patients were classified as the erythematous/ulcerative subtype, while the other 20 were classified as reticular subtype. Forty healthy volunteers (female/male: $21 / 19$; mean age: $42 \pm 15$ years old), which were age-sex-matched to the patients of erythematous/ulcerative group, were recruited as control group. All subjects were non-smokers who neither had any severe systemic disorders (such as diabetes mellitus, chronic hepatitis $\mathrm{C}$, etc.), nor any soft tissue lesions in the oral cavity. Subjects with periodontitis were excluded from the current study. And those with gingivitis were subjected to supragingival scaling first and the sample collection was performed after the inflammation was resolved. Moreover, none of the participants received any

\footnotetext{
${ }^{1}$ College of Stomatology, Chongqing Medical University, Chongqing, China; ${ }^{2}$ Chongqing Research Center for Oral Diseases and Biomedical Science, Chongqing, China and ${ }^{3}$ State Key Laboratory of Oral Diseases, West China Hospital of Stomatology, Sichuan University, Chengdu, China

Correspondence: Dr HX Dan, State Key Laboratory of Oral Diseases, West China Hospital of Stomatology, Sichuan University, No. 14, Section 3, Renmin South Road, Chengdu 610041, China. 
medical treatments within at least 90 days prior to the sample collection. This study was carried out under the approval and supervision of the Committee for the Use of Human Subjects in Research in Sichuan University (approval number: WCHSIEC-D-2008-00002); informed consents were obtained from all the participants.

\section{Collection of samples}

Blood samples were collected between 8:00 and 10:00 a.m. from subjects. The blood sample was allowed to clot for $30 \mathrm{~min}$ before the separation of the serum. Serum was separated from blood cells by centrifugation at $1000 \mathrm{~g}$ for $5 \mathrm{~min}$. Whole unstimulated saliva sample, which was paired with serum sample from each participant, was collected simultaneously as described in our previous study. ${ }^{15}$ After centrifugation at $3500 \mathrm{~g}$ for $20 \mathrm{~min}$, the clarified supernatant of the saliva was collected. All the samples were stored at $-80{ }^{\circ} \mathrm{C}$ for further use.

\section{Cytokines assays}

Serum and saliva samples were kept at room temperature for about $1 \mathrm{~h}$ to defrost completely before assays. IFN- $\gamma$ (sensitivity: $8 \mathrm{ng} \cdot \mathrm{L}^{-1}$ ) and IL-4 (sensitivity: $10 \mathrm{ng} \cdot \mathrm{L}^{-1}$ ) concentrations were determined in duplicate using enzyme-linked immunosorbent assay kits (R\&D Systems, Minneapolis, MN, USA). The standard curve range used in this study was 3.9-250 ng $\cdot \mathrm{L}^{-1}$ for IFN- $\gamma$ and $7.8-500 \mathrm{ng} \cdot \mathrm{L}^{-1}$ for IL-4. Data were read with a spectrophotometer (Sirio; Seac, Florence, Italy) at $450 \mathrm{~nm}$, the concentrations of IFN- $\gamma$ and IL- 4 in the samples were determined by extrapolating from the standard curve created by plotting the absorbance of the standards versus corresponding concentrations, and results were expressed as $\mathrm{ng} \cdot \mathrm{mL}^{-1}$.

\section{Statistical analysis}

Statistical Package for Social Science (SPSS 13.0 for Windows; SPSS Inc., Chicago, IL, USA) software was used to analyze the data. Results were expressed as mean \pm standard deviation. Two-sample (Student's) $t$-tests were used for pairwise comparison of OLP group and control group. One-way analysis of variance was used to compare the differences between the two OLP subtype groups and between the each OLP subtype group and the control group. Additionally, correlation of the IFN- $\gamma$ and IL-4 expression levels in serum and paired salivary samples in OLP patients was assessed by Pearson's correlation. $P<0.05$ was considered statistically significant.

\section{RESULTS}

\section{Serum levels of IFN- $\gamma$, IL-4 and IFN- $\gamma / \mathrm{IL}-4$ ratio}

As shown in Table 1 , serum IFN- $\gamma$ level of OLP patients was markedly lower than control group $(P<0.001)$. When OLP patients were divided into erythematous/ulcerative subgroup and reticular subgroup, IFN- $\gamma$ levels demonstrated the same tendency, compared with control group $(P<0.001 ; P=0.006$; Figure 1$)$, while there was no

\section{Table 1 Expression of IFN $-\gamma$ and IL-4 in serum in OLP patients}

\begin{tabular}{|c|c|c|c|}
\hline Items & $\mathrm{IFN}-\gamma /\left(\mathrm{ng} \cdot \mathrm{L}^{-1}\right)$ & $\mathrm{IL}-4 /\left(\mathrm{ng} \cdot \mathrm{L}^{-1}\right)$ & IFN- $\gamma / \mathrm{IL}-4$ \\
\hline $\operatorname{OLP}(n=60)^{\mathrm{a}}$ & $21.057 \pm 11.860 *$ & $20.545 \pm 3.482 *$ & $1.048 \pm 0.612 *$ \\
\hline Erythematous/ulcerative $(n=40)$ & $19.492 \pm 3.121^{* b}$ & $21.188 \pm 3.511^{*}$ & $0.925 \pm 0.606 *$ \\
\hline Reticular $(n=20)$ & $24.185 \pm 7.455^{*}$ & $19.259 \pm 3.121 *$ & $1.296 \pm 0.472 *$ \\
\hline Control $(n=40)$ & $31.935 \pm 10.867$ & $15.917 \pm 2.897$ & $2.130 \pm 1.013$ \\
\hline OLP vs. control & $P<0.001$ & $P<0.001$ & $P<0.001$ \\
\hline Erythematous/ulcerative OLP vs. control & $P<0.001$ & $P<0.001$ & $P<0.001$ \\
\hline Reticular OLP vs. control & $P=0.006$ & $P<0.001$ & $P<0.001$ \\
\hline Erythematous/ulcerative OLP vs. reticular OLP & $P=0.086$ & $P=0.042$ & $P=0.014$ \\
\hline
\end{tabular}

IFN, interferon; IL, interleukin; OLP, oral lichen planus.

$* P<0.05$ when compared with the control group.

${ }^{a}$ Mean value of OLP group were calculated based on the erythematous/ulcerative group and reticular group.

${ }^{\mathrm{b}}$ Seven of the samples had a value below the assay detection range, and their values were estimated by the regression equation of standard curve.

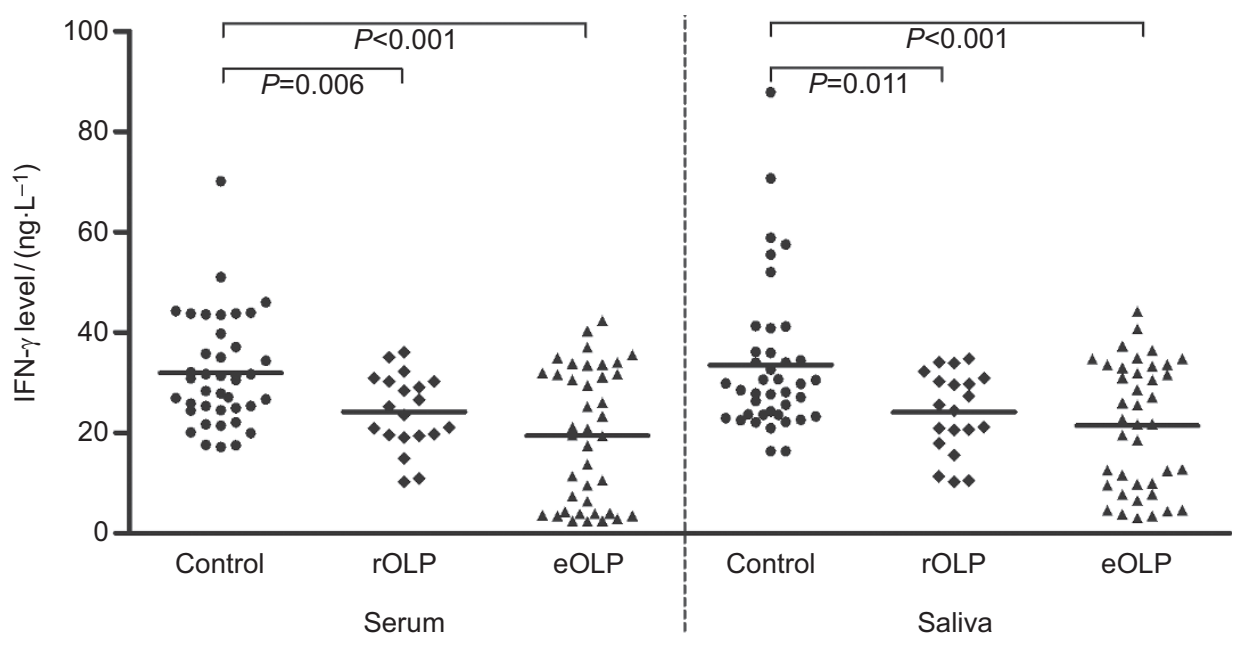

Figure 1 IFN- $\gamma$ levels of serum and saliva in OLP patients. OLP, oral lichen planus; rOLP, reticular oral lichen planus; eOLP, erythematous/ulcerative oral lichen planus. 
Table 2 Expression of IFN- $\gamma$ and IL-4 in saliva from OLP patients

\begin{tabular}{|c|c|c|c|}
\hline Items & $\mathrm{IFN}-\gamma /\left(\mathrm{ng} \cdot \mathrm{L}^{-1}\right)$ & $\mathrm{IL}-4 /\left(\mathrm{ng} \cdot \mathrm{L}^{-1}\right)$ & IFN- $\gamma /$ IL-4 \\
\hline $\operatorname{OLP}(n=60)^{\mathrm{a}}$ & $22.327 \pm 11.260 *$ & $21.564 \pm 3.422 *$ & $1.059 \pm 0.559 *$ \\
\hline Erythematous/ulcerative $(n=40)$ & $21.439 \pm 12.567 * b$ & $22.317 \pm 3.779 *$ & $0.983 \pm 0.606 *$ \\
\hline Reticular $(n=20)$ & $24.104 \pm 8.037 *$ & $20.059 \pm 1.863$ & $1.213 \pm 0.424^{*}$ \\
\hline Control $(n=40)$ & $33.517 \pm 14.968$ & $19.624 \pm 1.221$ & $1.728 \pm 0.821$ \\
\hline OLP vs. control & $P<0.001$ & $P<0.001$ & $P<0.001$ \\
\hline Erythematous/ulcerative OLP vs. control & $P<0.001$ & $P<0.001$ & $P<0.001$ \\
\hline Reticular OLP vs. control & $P=0.011$ & $P=0.282$ & $P=0.011$ \\
\hline Erythematous/ulcerative OLP vs. reticular OLP & $P=0.324$ & $P=0.003$ & $P=0.094$ \\
\hline
\end{tabular}

IFN, interferon; IL, interleukin; OLP, oral lichen planus.

$* P<0.05$ when compared with the control group.

${ }^{a}$ Mean value of OLP group were calculated based on the erythematous/ulcerative group and reticular group.

${ }^{\mathrm{b}}$ Two of the samples had a value below the assay detection range, and their values were estimated by the regression equation of standard curve.

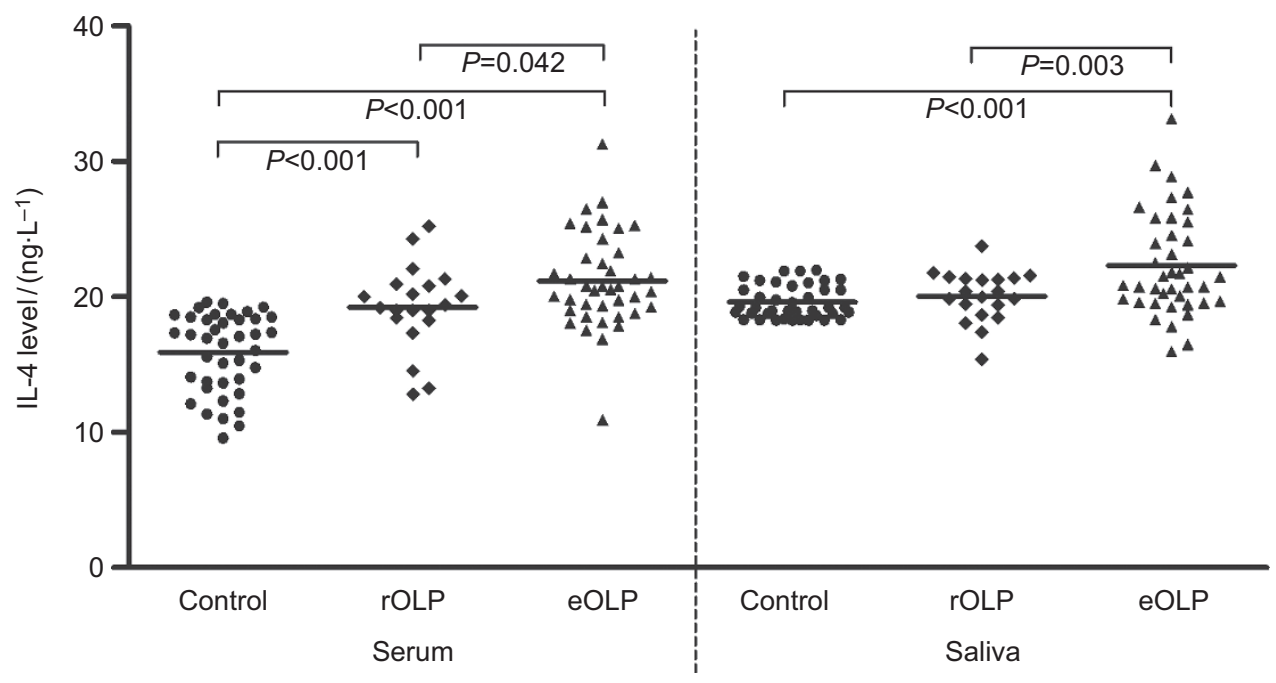

Figure 2 IL-4 levels of serum saliva in OLP patients. IL, interferon; OLP, oral lichen planus; rOLP, reticular oral lichen planus; eOLP, erythematous/ulcerative oral lichen planus.

significant difference of IFN- $\gamma$ level between the two different subtypes of OLP $(P=0.086)$.

On the contrary, serum IL-4 level of OLP patients was higher than control group $(P<0.001)$. IL-4 levels in erythematous/ulcerative subgroup and reticular subgroup demonstrated the same tendency compared with control group ( $P<0.001 ; P<0.001$; Figure 2$)$. Furthermore, the mean serum IL-4 level was higher in erythematous/ulcerative group than in reticular OLP group $(P=0.042)$.

Serum IFN- $\gamma /$ IL-4 ratio was lower in OLP group than control group $(P<0.001)$. Among subtypes of OLP, this ratio was lower in erythematous/ulcerative group than in reticular group $(P=0.014)$.

\section{Saliva levels of IFN- $\gamma$, IL-4 and IFN- $\gamma /$ IL-4 ratio}

As shown in Table 2, saliva IFN- $\gamma$ level of OLP patients was significantly lower than control group $(P<0.001)$, while there was no significant difference of IFN- $\gamma$ level between the two different subtypes of OLP $(P=0.324$; Figure 1$)$.

Saliva IL-4 level of OLP patients or patients with erythematous/ ulcerative OLP was higher than control group (both $P<0.001$; Figure 2). Furthermore, the mean saliva IL-4 level was higher in erythematous/ ulcerative group than in reticular OLP group $(P=0.003)$. There was no significant difference of IL-4 level between patients with reticular OLP and healthy controls ( $P=0.282)$.

Saliva IFN- $\gamma /$ IL-4 ratio was lower in OLP group than control group $(P<0.001)$, with no significant difference between two subtypes of $\operatorname{OLP}(P=0.094)$.

Correlations of IFN- $\gamma$ and IL-4 levels between serum and saliva Saliva levels of IFN- $\gamma$ and IL- 4 in OLP patients were significantly higher than their serum partners $(P=0.005$ and $P=0.007$, respectively). A significant positive correlation of cytokine levels between serum and saliva was found in OLP patients (Figure 3), either in the whole group (IFN- $\gamma$ : correlation coefficient $=0.954, P<0.001$; IL-4: correlation coefficient $=0.696, P<0.001$ ) or the erythematous/ulcerative subgroup $($ IFN- $\gamma$ : correlation coefficient $=0.985, P<0.001$; IL-4: correlation coefficient $=0.682, P<0.001)$ and reticular subgroup (IFN$\gamma$ : correlation coefficient $=0.797, P<0.001$; IL-4: correlation coefficient $=0.710, P<0.001)$. Meanwhile, IFN- $\gamma / \mathrm{IL}-4$ ratio in serum from OLP patient was also positively correlated with its saliva partner (whole OLP group: correlation coefficient $=0.923, P<0.001$; erythematous/ulcerative subgroup: correlation coefficient $=0.965, P<0.001$; reticular subgroup: correlation coefficient $=0.758, P<0.001$ ). 

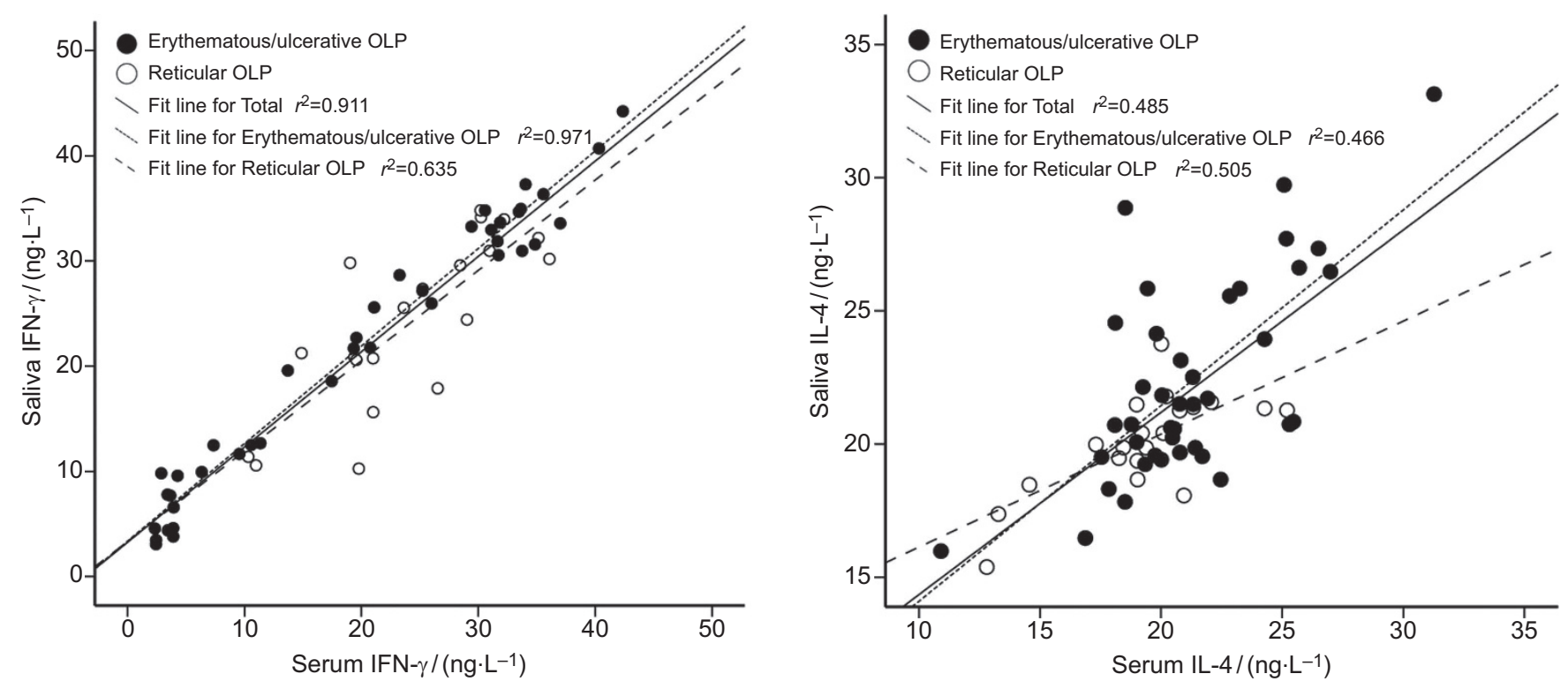

Figure 3 Correlations of cytokines levels between serum and saliva in OLP patients. OLP, oral lichen planus.

\section{DISCUSSION}

Cytokines but not cytokine-secreted cells drive and maintain the in vivo immune response. IFN- $\gamma$ and IL-4, the most characteristic Th1 and Th2 cytokines, respectively, regulate the T-cell differentiation and Th1/Th2 balance involved in physiological and pathological immune processes. IFN- $\gamma$ is involved in the maturation and activation of CD $8^{+}$ cytotoxic $\mathrm{T}$ cell and maintaining the expression of major histocompatibility class II molecules, thus participating in keratinocyte apoptosis and disease chronicity of OLP. ${ }^{16}$ IL-4, on the other hand, is necessary for Th2 cell differentiation and plays an important role in regulation of antibody production and humoral immune response. ${ }^{1}$ As IFN- $\gamma$ inhibits the expression of Th2 cytokines such as IL-4, and vice versa, the IFN- $\gamma / \mathrm{IL}-4$ cytokine ratio is considered to be a simple and direct indicator of Th1/Th2 balance. ${ }^{17}$ Being consistent with our previous salivary study, the present study demonstrated lower IFN- $\gamma$ but higher IL-4 expression levels in serum of OLP patient, with a lower IFN- $\gamma / \mathrm{IL}-4$ ratio when compared to healthy controls. Taken together, our data indicated an imbalance favoring Th2 predominance in OLP.

Although the precise etiology of OLP is still unknown, it has been widely accepted that immunological aberrances are very critical among the multiple etiological factors. ${ }^{9,18}$ The role of Th1/Th2 imbalance in the pathogenesis of OLP has been studied extensively in the past few years. Based on the expression of Th1 cytokines (IFN- $\gamma$ and tumor-necrosis factor- $\alpha$ ) but not IL- 4 and IL-10 by $\mathrm{T}$ cells in the lymphocytic infiltrate of OLP lesion and the pathological hypothesis of OLP that intra-epithelial Th1-activated autocytotoxic $\mathrm{CD} 8{ }^{+} \mathrm{T}$ cells trigger keratinocyte apoptosis, Sugerman et al. ${ }^{10,19}$ suggested that OLP was characterized by Th1 cytokine bias. However, simultaneous expression of Th1 and Th2 cytokines are detected in OLP local lesion and tissue transudates. ${ }^{13-14}$ Furthermore, saliva, serum and peripheral blood mononuclear cells from OLP patients present a mixed general expression profile of both Th1 and Th2 cytokines. ${ }^{11,14-15,20}$ There is no doubt that both Th1 and Th2 immune responses are involved in the development of OLP.

Calculating and comparing the ratio of Th1/Th2-related cytokine and transcription factor expression might provide a clue for the exploration of Th1/Th2 imbalance in OLP etiology. In our studies, as well as a study by Rhodus and colleagues, ${ }^{13,15}$ a Th2 skew in OLP patients was demonstrated. Nevertheless, in another study, in which the expression of specific transcription factors of Th1 and Th2 was examined, a predominant role of Th1-type immune response was implicated. ${ }^{21}$ Yet in another study, which calculated the IFN- $\gamma /$ IL-4 ratio in lesional tissue and unstimulated whole saliva, the expression of both cytokines were elevated and the IFN- $\gamma / \mathrm{IL}-4$ ratio showed no significant difference in OLP patients and healthy controls. These controversies might partly be caused by the difference of patients, research methods or sample sizes, but it also implied that there might be some mechanisms, such as the role of regulatory $\mathrm{T}$ cells ${ }^{22}$ and new subsets of Th cells, ${ }^{23}$ in the etiology of OLP other than Th1/Th2 imbalance.

OLP has various clinical forms without accepted consensual criteria for the evaluation of severity. Patients are usually evaluated according to the clinical and pathological manifestations. ${ }^{24}$ Previous investigations indicated that the erythematous/ulcerative subtype seemed to cause more serious symptoms such as pain and bleeding, and be at risk for future malignant transformation compared with reticular subtype. ${ }^{25}$ In the current study, though the expression of both cytokines was significantly different in OLP patients and healthy controls, only IL-4 level (both in serum and in saliva) showed significant difference in between two subtypes of OLP. These results indicated that IL-4 might be detrimental for OLP clinical outcome and a fine biomarker reflecting the severity of OLP.

There were strong correlations of IFN- $\gamma$ and IL-4 levels and ratio between serum and saliva from OLP patients. It suggested that cytokine expression in OLP was consistent in saliva and serum, detection of salivary cytokines could reflect systemic cytokine expression profile in OLP. Furthermore, we observed that the salivary concentrations of the cytokines from patients with OLP were higher than their serum partners, probably due to production of cytokines from the inflammatory infiltration and keratinocytes in OLP lesion and/or dysfunction of the structural barriers in oral mucosa. It was consistent with the study by Zhang et al. ${ }^{26}$ With the advantages of easy access and noninvasive collection by individuals with modest training, whole saliva 
provides a cost-effective approach for the repetitious disease monitoring and the screening of large populations in studies of systemic diseases and especially oral diseases. It might be a better routine substitute for other OLP test means to reflect the severity of OLP by detecting the salivary IL-4 level. ${ }^{27}$

It is necessary to note, limited by the detection range of the enzymelinked immunosorbent assay kit, some IFN- $\gamma$ detected values in the erythematous/ulcerative OLP group were below the detection range and estimated by the regression equation of standard curve, which might not be accurate. A more sensitive assay should be need for the further studies.

In general, our current study, together with our previous study, identified the Th2 cytokine-predominant immune imbalance in OLP and indicated that IL-4 level might be in association with severity of OLP.

\section{ACKNOWLEDGEMENTS}

This work was supported by grants from the National Natural Science Foundation of China (No. 30930100, 81072218, 81200791, 81321002 and 81300882), the Doctoral Program of the Ministry of Education of China (No. 20110181110055 and 20120181120011) and Natural Science Foundation of Chongqing (No. cstc2013jcyjA10042). We appreciate Dr Shu Yang and Dr Keli Chen from West China College of Public Health, Sichuan University, China, for their help with statistical analysis. Publication of this manuscript is supported by Open Fund of State Key Laboratory of Oral Diseases, Sichuan University.

1 Neurath MF, Finotto S, Glimcher LH. The role of Th1/Th2 polarization in mucosal immunity. Nat Med 2002; 8(6): 567-573.

2 Rengarajan J, Szabo SJ, Glimcher LH. Transcriptional regulation of Th1/Th2 polarization. Immunol Today 2000; 21(10): 479-483.

3 Robinson DS, Hamid Q, Ying S et al. Predominant TH2-like bronchoalveolar Tlymphocyte population in atopic asthma. N Engl J Med 1992; 326(5): 298-304.

4 Fuss IJ, Neurath M, Boirivant M et al. Disparate CD4 ${ }^{+}$lamina propria (LP) lymphokine secretion profiles in inflammatory bowel disease. Crohn's disease LP cells manifest increased secretion of IFN-gamma, whereas ulcerative colitis LP cells manifest increased secretion of IL-5. J Immunol 1996; 157(3): 1261-1270.

5 Plevy SE, Landers CJ, Prehn J et al. A role for TNF-alpha and mucosal T helper-1 cytokines in the pathogenesis of Crohn's disease. J Immunol 1997; 159(12): 62766282.

6 Ghoreschi K, Thomas P, Breit S et al. Interleukin-4 therapy of psoriasis induces Th2 responses and improves human autoimmune disease. Nat Med 2003; 9(1): 40-46.

7 Al-Hashimi I, Schifter M, Lockhart PB et al. Oral lichen planus and oral lichenoid lesions: diagnostic and therapeutic considerations. Oral Surg Oral Med Oral Pathol Oral Radiol Endod 2007; 103(3): S25-S31.

8 Sugerman PB, Savage NW. Oral lichen planus: causes, diagnosis and management. Aust Dent J 2002; 47(4): 290-297.
9 Sugerman PB, Savage NW, Walsh LJ et al. The pathogenesis of oral lichen planus. Crit Rev Oral Biol Med 2002; 13(4): 350-365.

10 Khan A, Farah CS, Savage NW et al. Th1 cytokines in oral lichen planus. J Oral Pathol Med 2003; 32(2): 77-83.

11 Karagouni EE, Dotsika EN, Sklavounou A. Alteration in peripheral blood mononuclear cell function and serum cytokines in oral lichen planus. J Oral Pathol Med 1994; 23(1): 28-35.

12 Ichimura M, Hiratsuka K, Ogura $\mathrm{N}$ et al. Expression profile of chemokines and chemokine receptors in epithelial cell layers of oral lichen planus. J Oral Pathol Med 2006; 35(3): 167-174.

13 Rhodus NL, Cheng B, Ondrey F. Th1/Th2 cytokine ratio in tissue transudates from patients with oral lichen planus. Mediators Inflamm 2007; 2007: 19854.

14 Tao XA, Li CY, Rhodus NL et al. Simultaneous detection of IFN-gamma and IL-4 in lesional tissues and whole unstimulated saliva from patients with oral lichen planus. J Oral Pathol Med 2008; 37(2): 83-87.

15 Liu WZ, Dan HX, Wang Z et al. IFN-gamma and IL-4 in saliva of patients with oral lichen planus: a study in an ethnic Chinese population. Inflammation 2009; 32(3): 176 181.

16 Lodi G, Scully C, Carrozzo M et al. Current controversies in oral lichen planus: report of an international consensus meeting. Part 1. Viral infections and etiopathogenesis. Oral Surg Oral Med Oral Pathol Oral Radiol Endod 2005; 100(1): 40-51.

17 Gómez D, Correa PA, Gómez LM et al. Th1/Th2 cytokines in patients with systemic lupus erythematosus: is tumor necrosis factor alpha protective? Semin Arthritis Rheum 2004; 33(6): 404-413.

18 Crincoli V, Di Bisceglie MB, Scivetti M et al. Oral lichen planus: update on etiopathogenesis, diagnosis and treatment. Immunopharmacol Immunotoxicol 2011; 33(1): 11-20.

19 Simark-Mattsson C, Bergenholtz G, Jontell M et al. Distribution of interleukin-2,-4, -10 , tumour necrosis factor-alpha and transforming growth factor-beta mRNAs in oral lichen planus. Arch Oral Biol 1999; 44(6): 499-507.

20 Yamamoto T, Yoneda K, Ueta E et al. Serum cytokines, interleukin-2 receptor, and soluble intercellular adhesion molecule-1 in oral disorders. Oral Surg Oral Med Oral Pathol 1994; 78(6): 727-735.

21 Lu R, Zhou G, Du GF et al. Expression of T-bet and GATA-3 in peripheral blood mononuclear cells of patients with oral lichen planus. Arch Oral Biol 2011; 56(5): 499-505.

22 Tao XA, Xia J, Chen XB et al. FOXP3 ${ }^{+}$T regulatory cells in lesions of oral lichen planus correlated with disease activity. Oral Dis 2010; 16(1): 76-82.

23 Tesmer LA, Lundy SK, Sarkar S et al. Th17 cells in human disease. Immunol Rev 2008; 223: 87-113.

24 Eisen D, Carrozzo M, Bagan Sebastian JV et al. Number V oral lichen planus: clinical features and management. Oral Dis 2005; 11(6): 338-349.

25 Eisen $D$. The clinical features, malignant potential, and systemic associations of oral lichen planus: a study of 723 patients. J Am Acad Dermatol 2002; 46(2): 207-214.

26 Zhang YY, Lin M, Zhang ST et al. NF-kappa B-dependent cytokines in saliva and serum from patients with oral lichen planus: a study in an ethnic Chinese population. Cytokine 2008; 41(2): 144-149.

27 Kaufman E, Lamster IB. The diagnostic applications of saliva-a review. Crit Rev Oral Biol Med 2002; 13(2): 197-212.

This work is licensed under a Creative Commons Attribution-NonCommercial-NoDerivative Works 3.0 Unported License. To view a copy of this license, visit http:// creativecommons.org/licenses/by-nc-nd/3.0 